\title{
Elastosis during airway wall remodeling explains multiple co-existing instability patterns
}

\author{
Mona Eskandari ${ }^{\mathrm{a}}$, Ali Javili ${ }^{\mathrm{b}}$, Ellen Kuhla ${ }^{\mathrm{a}, \mathrm{c}, \mathrm{d}}$ \\ ${ }^{a}$ Department of Mechanical Engineering, Stanford University, Stanford, CA 94305, USA \\ ${ }^{b}$ Department of Civil and Environmental Engineering, Stanford University, Stanford, CA 94305, USA \\ ${ }^{c}$ Department of Bioengineering, Stanford University, Stanford, CA 94305, USA \\ ${ }^{d}$ Department of Cardiothoracic Surgery, Stanford University, Stanford, CA 94305, USA \\ email: ekuhl@stanford.edu, phone: +1.650.450.0855,fax: +1.650.725.1587, URL: http:/biomechanics.stanford.edu, corresponding author
}

\begin{abstract}
Living structures can undergo morphological changes in response to growth and alterations in microstructural properties in response to remodeling. From a biological perspective, airway wall inflammation and airway elastosis are classical hallmarks of growth and remodeling during chronic lung disease. From a mechanical point of view, growth and remodeling trigger mechanical instabilities that result in inward folding and airway obstruction. While previous analytical and computational studies have focused on identifying the critical parameters at the onset of folding, few have considered the post-buckling behavior. All prior studies assume constant microstructural properties during the folding process; yet, clinical studies now reveal progressive airway elastosis, the degeneration of elastic fibers associated with a gradual stiffening of the inner layer. Here, we explore the influence of temporally evolving material properties on the post-bifurcation behavior of the airway wall. We show that a growing and stiffening inner layer triggers an additional subsequent bifurcation after the first instability occurs. Evolving material stiffnesses provoke failure modes with multiple co-existing wavelengths, associated with the superposition of larger folds evolving on top of the initial smaller folds. This phenomenon is exclusive to material stiffening and conceptually different from the phenomenon of period doubling observed in constant-stiffness growth. Our study suggests that the clinically observed multiple wavelengths in diseased airways are a result of gradual airway wall stiffening. While our evolving material properties are inspired by the clinical phenomenon of airway elastosis, the underlying concept is broadly applicable to other types of remodeling including aneurysm formation or brain folding.
\end{abstract}

Keywords: Instability; folding; airway; elastosis; growth and remodeling; finite element analysis

\section{Motivation}

Millions of people worldwide suffer from progressive airflow obstruction. Long-term lung diseases such as chronic obstructive pulmonary disease and asthma are of significant concern, they cost beyond 66 billion dollars $[8,69]$ and rank fourth as the leading cause of death [55]. The sheer number of fatalities motivates the need to understand the physiology of chronic pulmonary disease and inspires progress towards creating interventions, which would improve the quality of life of millions of people [3].

Figure 1 contrasts histological images of healthy and asthmatic airways. The healthy airway has thin layers and many regular folds with short wavelengths. The asthmatic airway has an engorged thickness and fewer, deeper folds with long wavelengths $[29,38]$. The asthmatic whole-airway image highlights three deep, long-wavelength folds superposed onto the regularly folded airway wall. The number of folds is of high clinical significance: few folds with large wavelengths move inwards and completely obstruct the lumen, whereas numerous folds with short wavelength, as seen in healthy subjects, leave the airway unobstructed $[42,68]$. This explains why the fold number has been proposed as a quantitative surrogate measure of airway obstruction, as supported by clinical observations [18].
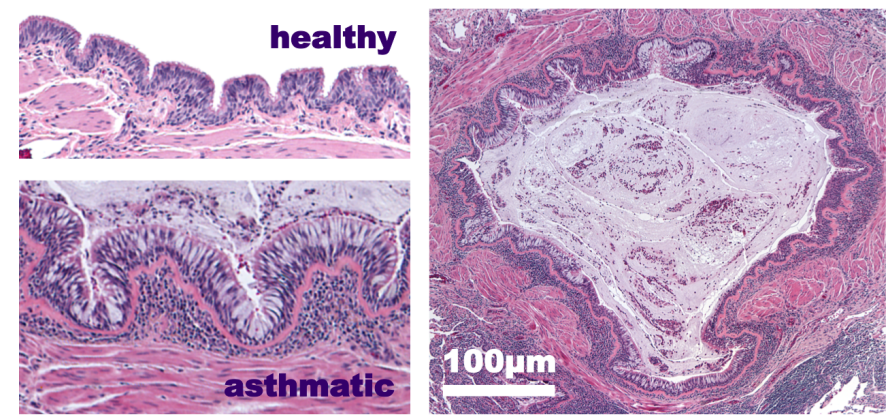

Figure 1: Histological images of healthy and asthmatic airways. In contrast to the healthy airway (top left), the asthmatic airway (bottom left) displays larger, deeper folds with thickened, inflamed layers. The whole-airway image (right) illustrates the characteristic configuration of multiple small, regular folds and a co-existing instability pattern of three superposed large, deep folds.

The common nomenclature of the airway wall groups the epithelium, the lamina propria, and the basement membrane into the innermost airway lining called the mucosa, enclosed by the softer, thicker, loosely connected elastin and collagen rich submucosal layer $[5,38]$. The distinct properties of the mucosa and submucosa motivate their modeling as a bi-layered sys- 


\section{healthy airway wall}

epithelium

basement membrane

elastic fibrous network

smooth muscle

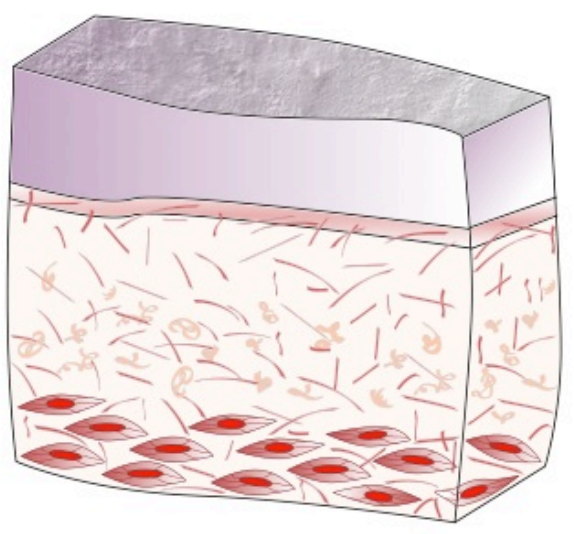

elastosis in diseased airway wall

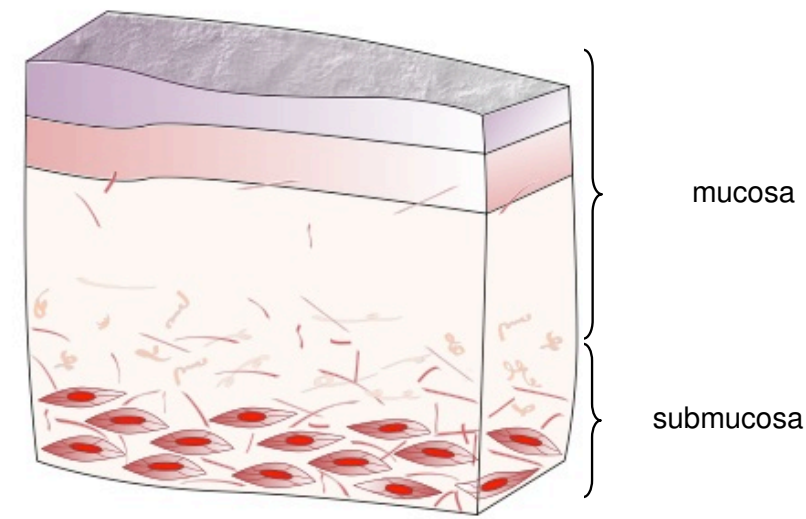

oxytalanic and elauninic fibers

elastic fibers

Figure 2: Schematic illustration of healthy airway wall (left) and elastosis in diseased airway wall (right). The collagenous and stiff mucosal layer, composed of the epithelium and basement membrane, sits on top of the compliant submucosal substrate. In prior studies, airway wall obstruction was attributed exclusively to the thickening of the basement membrane, a growing stiff layer on a soft substrate. Previous studies neglect the progressive loss and fragmentation of the elastin network associated with a gradual stiffening of the mucosal layer. Here, we model airway obstruction as the combined phenomenon of growth and remodeling with a a gradually growing and stiffening layer on top of a soft substrate.

tem with a stiff layer on a soft substrate $[33,35]$. Both layers are surrounded by smooth muscle, which is thicker in diseased airways and applies increased external contractile force, which may cause the airway wall to collapse $[34,48,58]$. While initial studies have considered smooth muscle as the major cause of airway obstruction $[63,33,68]$, recent evidence suggests that airway instabilities may be initiated by airway inflammation and an influx of cells triggered by inhaled toxins and pollutants $[14,45,53]$. The latter phenomenon-modeled by the theory of finite growth [60]-has been the focus of several recent analytical and computational studies.

Growth of the inner mucosal lining generates compressive stresses causing instabilities that buckle into folds [19, 54, 70]. Given the variability in the structure of diseased airways versus healthy airways [21, 41], several models have established the role of the thickness ratio and the elasticity ratio between the mucosal and submucosal layers to dictate pattern formation $[33,67]$. Increased thickness of the mucosa, submucosa, and stiffness ratios provoke increased wavelengths and fewer folds. Rigorous relationships between these ratios and the number of folds have been established for circular, elliptical, and rectangular geometries $[6,17,22]$ and even for irregular patient-specific geometries [23].

To predict buckling instabilities in the diseased airway, previous analytical and computational models assume that the structural integrity of the airway wall remains constant, while clinical findings indicate otherwise [4, 16]. Chronic lung disease is typically associated with airway elastosis, the gradual remodeling of the airway wall via fiber reorientation and altered material properties [9, 40, 71]. Diseased airways exhibit a degeneration of the elastic fibers necessary for recoil during exhalation. Elastosis results in a marked loss of airway wall compliance $[15,18]$. Figure 2 illustrates the fragmentation of these fibers during airway wall remodeling and the associated stiffening of the mucosal layer [49].

Here we model chronic lung disease using continuum theories of superposed growth and remodeling. Continuum modeling is a powerful tool to explore the pre- and post-buckling regime of a remodeling airway segment with temporally evolving size and stiffness properties. Increasing the mucosal volume accounts for growth while increasing the mucosal stiffness accounts for remodeling [65]. The resulting folding patterns of combined growth and remodeling provide a non-trivial explanation of the pathophysiological folding patterns observed in the clinical setting. Our simulations predict multiple co-existing instability modes and agree qualitatively and quantitatively with histological findings in chronic lung disease.

In the following sections, we illustrate how we implement airway growth to mimic inflammation and airway remodeling to mimic elastosis within a finite element setting. We contrast the results from constant-stiffness to evolving stiffness and highlight the variety of emerging folding patterns in response to varying stiffening rates. We demonstrate that our results are independent of the underlying geometry and cannot be explained as a trivial after-effect of continuing growth alone.

\section{Methods}

To model the impact of increasing stiffness and loss of compliance on the emerging folding pattern, we apply growth with temporally varying material elastic response using a continuum mechanics approach. We discretize the idealized cylindrical airway wall geometry and employ a finite element analysis with growth and remodeling as a user-defined material subroutine.

\subsection{Airway inflammation: Growth and changes in volume}

To model airway inflammation, we adapt the kinematics of finite growth and allow the airway wall to gradually increase 


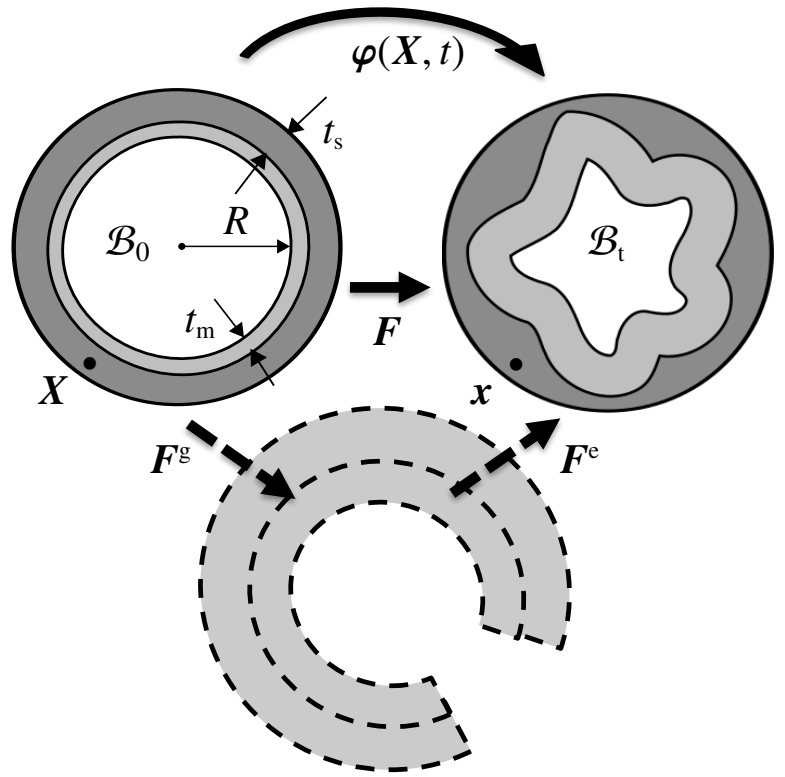

Figure 3: Geometric representation of two-dimensional airway wall model and kinematics of growth. Thickness of mucosa $t_{\mathrm{m}}$ and thickness of the submucosa $t_{\mathrm{S}}$ are non-dimensionalized by radius $R$. The undeformed configuration $\mathcal{B}_{0}$ is mapped to the deformed configuration $\mathcal{B}_{\mathrm{t}}$ through $\varphi(X, t)$. Growth is represented through the multiplicative decomposition of the deformation gradient $\boldsymbol{F}$ into an elastic tensor $\boldsymbol{F}^{\mathrm{e}}$ and a growth tensor $\boldsymbol{F}^{\mathrm{g}}$

in volume $[25,28]$. As shown in Figure 3, we characterize the mapping from a point $\boldsymbol{X}$ in the initial healthy configuration $\mathcal{B}_{0}$ to the corresponding point $\boldsymbol{x}$ in the current diseased configuration $\mathcal{B}_{t}$ at time $t$ by the deformation map $\boldsymbol{x}=\boldsymbol{\varphi}(X, t)$. Line elements $\mathrm{d} \boldsymbol{X}$ and $\mathrm{d} \boldsymbol{x}$ relate the initial and current tangent spaces $T \mathcal{B}_{0}$ and $T \mathcal{B}_{\mathrm{t}}$ through the deformation gradient $\boldsymbol{F}=\nabla_{X} \boldsymbol{\varphi}$ as $\mathrm{d} \boldsymbol{x}=\boldsymbol{F} \cdot \mathrm{d} \boldsymbol{X}$. We multiplicatively decompose the deformation gradient $\boldsymbol{F}$ into an elastic part $\boldsymbol{F}^{\mathrm{e}}$ and a growth part $\boldsymbol{F}^{\mathrm{g}}$ $[2,60,73]$,

$$
\boldsymbol{F}=\boldsymbol{F}^{\mathrm{e}} \cdot \boldsymbol{F}^{\mathrm{g}} .
$$

The Jacobian $J=\operatorname{det}(\boldsymbol{F})$ defines the change in airway wall volume, such that infinitesimal volume elements $\mathrm{d} V$ and $\mathrm{d} v$ are related through $\mathrm{d} v=J \mathrm{~d} V$. Similarly, we can divide the total volume change $J$ into a reversible elastic volume change $J^{\mathrm{e}}$ and an irreversible growth volume change $J^{\mathrm{g}}$,

$$
J=J^{\mathrm{e}} J^{\mathrm{g}} .
$$

We assume that the airway wall grows morphogenetically and isotropically [24, 32]. We prescribe the growth tensor $\boldsymbol{F}^{\mathrm{g}}$ as the identity tensor $\boldsymbol{I}$ scaled by the growth multiplier $\vartheta$ [27],

$$
\boldsymbol{F}^{\mathrm{g}}=\vartheta \boldsymbol{I} .
$$

We propose that the airway wall grows linearly in time at a constant growth rate $\dot{\vartheta}=G_{\vartheta}$,

$$
\vartheta=\vartheta_{0}+G_{\vartheta} t \quad \text { with } \quad G_{\vartheta}=\frac{\vartheta_{\infty}-\vartheta_{0}}{t_{\infty}-t_{0}}
$$

where $\vartheta_{0}=1$ defines the initial ungrown state at $t_{0}$ and $\vartheta_{\infty}$ defines the maximum growth at $t_{\infty}$, with $t_{0} \leq t \leq t_{\infty}$. We can now explicitly express the elastic tensor $\boldsymbol{F}^{\mathrm{e}}=\boldsymbol{F} \cdot \boldsymbol{F}^{\mathrm{g}-1}$ with $\boldsymbol{F}^{\mathrm{g}-1}=\boldsymbol{I} / \vartheta$ as

$$
\boldsymbol{F}^{\mathrm{e}}=\boldsymbol{F} / \vartheta
$$

the elastic Jacobian $J^{\mathrm{e}}=J / J^{\mathrm{g}}$ with $J^{\mathrm{g}}=\vartheta^{3}$ as

$$
J^{\mathrm{e}}=J / \vartheta^{3}
$$

and the elastic left Cauchy-Green deformation tensor $\boldsymbol{b}^{\mathrm{e}}=\boldsymbol{F}^{\mathrm{e}}$. $\boldsymbol{F}^{\mathrm{et}}$ as

$$
\boldsymbol{b}^{\mathrm{e}}=\boldsymbol{F} \cdot \boldsymbol{F}^{\mathrm{t}} / \vartheta^{2}
$$

For simplicity, we characterize the airway wall through the Helmholtz free energy function for an isotropic, hyperelastic Neo-Hookean material,

$$
\psi=\frac{1}{2} \mu\left[\bar{I}_{1}^{\mathrm{e}}-3\right]+\frac{1}{2} k\left[J^{\mathrm{e}}-1\right]^{2},
$$

which has an isochoric and volumetric part and depends on two invariants, the Jacobian $J^{\mathrm{e}}$ and the first deviatoric invariant $\bar{I}_{1}^{\mathrm{e}}=\overline{\boldsymbol{b}}^{\mathrm{e}}: \boldsymbol{I}$, where $\overline{\boldsymbol{b}}^{\mathrm{e}}=\overline{\boldsymbol{F}}^{\mathrm{e}} \cdot \overline{\boldsymbol{F}}^{\mathrm{e}}=\left(J^{\mathrm{e}}\right)^{-2} \boldsymbol{b}^{\mathrm{e}}$. The penalty parameter $k$ enforces incompressibility and the Lamé constant $\mu$ characterizes the stiffness of the mucosa or submucosal layer $[31,62]$.

\subsection{Airway elastosis: Remodeling and changes in stiffness}

To model airway elastosis, we adapt the concept of remodeling [65], and gradually increase the stiffness of the inner mucosal layer, while the stiffness of the outer submucosa remains constant. In chronic lung disease, inflammation or growth and elastosis or remodeling occur at an unknown rate $[22,56]$. For simplicity, we assume that the mucosal layer stiffens linearly in time at a constant stiffening rate $\dot{\mu}=G_{\mu}$,

$$
\mu=\mu_{0}+G_{\mu} t \quad \text { with } \quad G_{\mu}=\frac{\mu_{\infty}-\mu_{0}}{t_{\infty}-t_{0}}
$$

where $\mu_{0}=1$ defines the initial stiffness at $t_{0}$ and $\mu_{\infty}$ defines the maximum stiffness at $t_{\infty}$, with $t_{0} \leq t \leq t_{\infty}$ [51, 52]. The evolving stiffness $\mu$ enters the isochoric part of the free energy (8). As a natural consequence of mucosal stiffening, the stiffness ratio between the mucosal and submucosal layers $\beta=\mu_{\mathrm{m}} / \mu_{\mathrm{s}}$ is no longer constant. It evolves as remodeling progresses and increases linearly in time,

$$
\beta=\frac{\mu_{\mathrm{m}}}{\mu_{\mathrm{s}}}=\beta_{0}+G_{\beta} t \quad \text { with } \quad G_{\beta}=\frac{\beta_{\infty}-\beta_{0}}{t_{\infty}-t_{0}},
$$

where $\beta_{0}=\mu_{\mathrm{m}, 0} / \mu_{\mathrm{s}}$ is this initial stiffness contrast of the healthy airway wall and $\beta_{\infty}=\mu_{\mathrm{m}, \infty} / \mu_{\mathrm{s}}$ is the stiffness contrast of the diseased airway wall. We will explore different scenarios, airway growth with and without superposed remodeling at varying stiffening rates $G_{\mu}$.

\subsection{Computational model}

We implement growth and remodeling as a user-defined subroutine UHYPER within the non-linear finite element solver Abaqus/Standard version $6.13[1,26]$. Within the subroutine, we calculate the free energy along with its derivatives with respect to the invariants $\bar{I}_{1}^{\mathrm{e}}$ and $J^{\mathrm{e}}$ [23]. We represent growth $\vartheta$ 
and remodeling $\beta=\mu_{\mathrm{m}} / \mu_{\mathrm{s}}$ through internal variables, which we store locally at the integration point level. Growth affects the free energy (8) through the elastic Jacobian $J^{\mathrm{e}}=J / \vartheta^{3}$ in the volumetric term $\frac{1}{2} k\left[J^{\mathrm{e}}-1\right]^{2}$ and remodeling affects the free energy through the stiffness $\mu$ in the isochoric term $\frac{1}{2} \mu\left[\bar{I}_{1}^{\mathrm{e}}-3\right]$. Following previous work, we assume that the mucosal layer grows and stiffens at a much faster rate than the underlying submucosa. We thus model the airway wall as a growing, stiffening inner lining surrounded by a non-growing, non-stiffening elastic substrate [37, 45].

We model the airway as a cylindrical system, and additionally analyze cuboid systems for comparison. For both, we assume incompressibility using a Poisson's ratio of 0.4995 and chose the boundary conditions to mimic a plane strain state. While plane strain conditions appear natural for long cylindrical structures, they remain a simplification and might neglect important longitudinal effects, which could affect the overall folding pattern $[7,20]$. However, our previous simulations with three-dimensional, personalized airway wall segments predict comparable folding patterns for different geometries and suggest that our analysis is rather insensitive to the effects of the third dimension [23].

Figure 3 illustrates the airway wall geometry with the thicknesses $t_{\mathrm{m}}$ of the mucosa and $t_{\mathrm{s}}$ of the submucosa, which we nondimensionalize by dividing them by the radius $R$ [23, 45, 67]. To create rectangular geometries for comparison, we take the unrolled circumference to match the length of the rectangle. We parameterize the model in terms of the thickness ratios $t_{\mathrm{m}} / R$ and $t_{\mathrm{S}} / R$ and the time-evolving stiffness ratio between the mucosa and submucosal layers $\beta=\mu_{\mathrm{m}} / \mu_{\mathrm{s}}$.

We perform mesh refinement studies to confirm convergence and select a sufficiently refined mesh for all further simulations. We discretize the geometry with 8-noded linear hybrid hexahedral C3D8H elements with three elements across the mucosa, 21 elements across the submucosa, and 240 elements along the circumference. For the cylindrical model, we applied Dirichlet boundary conditions at the outer submucosal layer. For the rectangular model, we apply Dirichlet boundary conditions at the bottom and both sides, but allow all boundary nodes to slide freely along the edge. We adopt an automatic time step size to ensure quadratic convergence of the global Newton Raphson iteration method.

To trigger instabilities, we apply a random perturbation between $0 \%$ and $1 \%$ of the scaled mucosal thickness $t_{\mathrm{m}} / R$ to the nodes of the inner mucosa surface. We repeat each random simulation multiple times to verify the reproducibility of the evolving instability pattern. While ridges and valleys form at different locations along the circumference, the different folding patterns are merely rotations of the circular pattern and translations of the rectangular pattern. We confirm that the computationally simulated initial instability pattern agrees with the analytically predicted instability mode [57]. Unlike in previous studies $[6,36,46]$, we did not have to prescribe specific folding patterns: our emerging instability modes are independent of the random perturbation and the folds are genuine results of the selected geometric and material parameters.
We explore various geometries along with varying initial stiffness contrasts, varying stiffening rates, and varying growth rates, similar to recent studies [11, 22, 45]. We focus on inner layer growth and analyze how inner layer stiffening influences the initiation and progression of instabilities to understand the role of elastosis and mucosal stiffening in pattern formation and airway obstruction.

\section{Results}

\subsection{Airway wall growth with and without remodeling}

Figure 4 compares airway wall growth with and without remodeling, or rather folding at a constant-stiffness and folding at a gradually evolving stiffness. In both cases, a similar preferred buckling mode emerges as a function of the mucosa and submucosa thickness and stiffness, $t_{\mathrm{m}} / R, t_{\mathrm{s}} / R$, and $\mu_{\mathrm{m}} / \mu_{\mathrm{s}}$. The folded configuration moves progressively inwards to ultimately obstruct the airway lumen $[42,68]$.
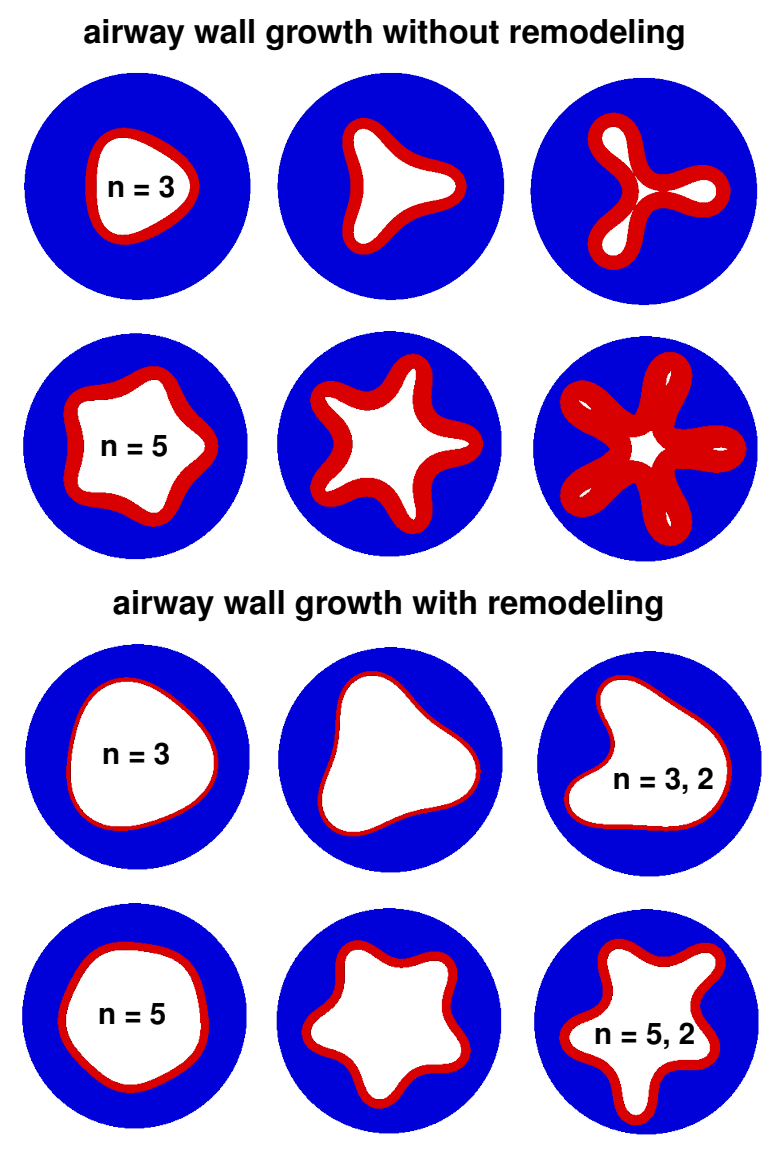

Figure 4: Airway wall growth without remodeling and with remodeling. Constant material properties trigger radially symmetric instabilities with folds that progressively move inwards and obstruct the lumen (top). Evolving material properties provoke secondary bifurcations and trigger non-symmetric instabilities (bottom). Previous studies are limited to regular constant-stiffness folding, while clinical observations suggest that airway wall remodeling involves stiffening and more complex folding patterns. First number indicates initial failure mode, second number indicates superposed secondary failure mode.

To date, folding has exclusively been attributed to growth of the inner layer or pressure of the outer layer. For constantstiffness instabilities, the initial failure mode is circularly 
symmetric, regular, and of a single wavelength. However, for evolving-stiffness instabilities, this initial buckling pattern is not the only failure mode. If stiffness alterations are significant, a subsequent bifurcation occurs before the folds can contact. The secondary instability is non-symmetric, irregular, and of multiple co-existing wavelengths. Figure 4 shows initial failure modes with $n=3$ and $n=5$ folds and, for evolving stiffnesses, additional superposed modes with $n=2$ folds.
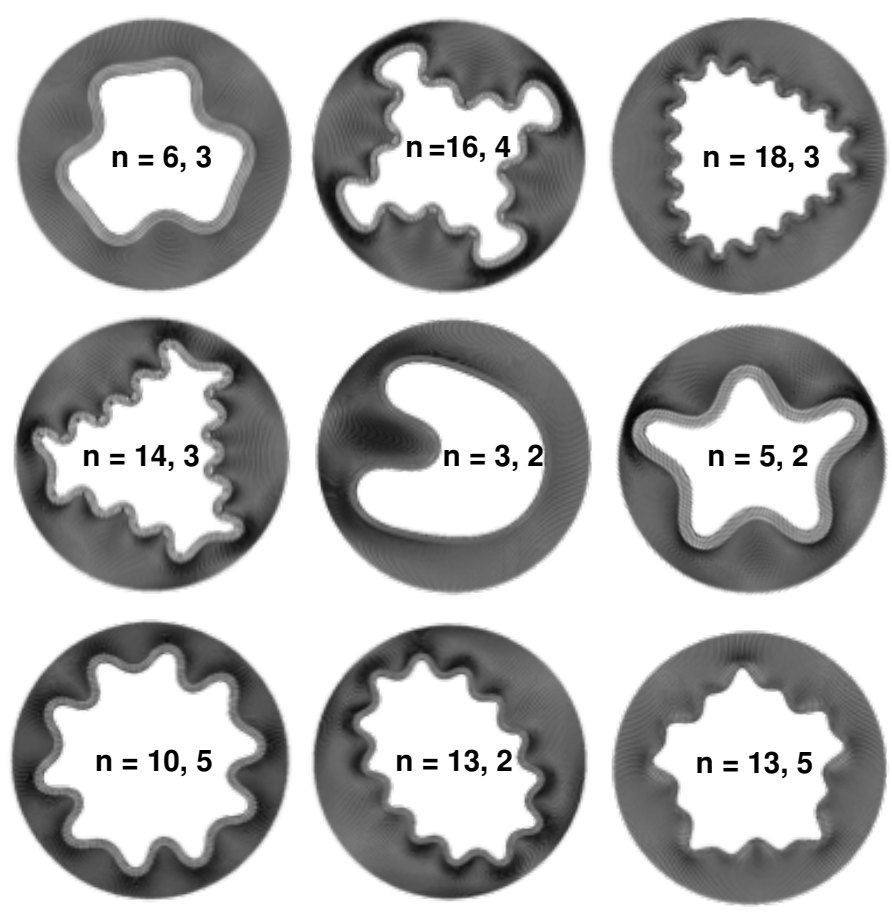

Figure 5: Examples of richer instability patterns arising during combined airway wall growth and remodeling. The first bifurcation is associated with a circularly symmetric, regular instability of a single wavelength. Airway elastosis and an altered stiffness contrast provoke secondary bifurcations and trigger non-symmetric, irregular instabilities characterized by multiple co-existing wavelengths. First number indicates initial failure mode, second number indicates superposed secondary failure mode.

\subsection{Variety of co-existing instability modes}

Figure 5 illustrates different folding instabilities for varying $t_{\mathrm{m}} / R, t_{\mathrm{s}} / R$, and $\mu_{\mathrm{m}} / \mu_{\mathrm{s}}$ parameters with gradually increasing stiffness contrast $\mu_{\mathrm{m}} / \mu_{\mathrm{s}}$. Initial failure modes vary between $\mathrm{n}=3,5,6,10,13,14,16$, and 18 folds with superposed modes with $\mathrm{n}=2,3,4$, and 5 folds.

Airway remodeling with coinciding growth generates richer, non-symmetric, more complex instability modes. Constantstiffness growth alone can generate a single unique failure mode, and, upon further growth, possibly further bifurcate into regular period doubling and period quadrupling [13]. However, growth alone cannot generate these unique, irregular folding patterns. In each example, an initial short-wavelength mode occurs and is later superposed by a long-wavelength mode associated with the increased stiffness contrast. Figure 5 illustrates the rich interplay of multiple co-existing instability pat- terns, which may even partly unfold and flatten out to accommodate the subsequent preferred mode. The superposition of multiple co-existing instability models agrees with the histological whole-airway image of Figure 1.

\subsection{Stiffening rate impacts instability mode}

Figure 6 illustrates selected folding patterns for different stiffness ratios and stiffening rates over the same period of growth. Overlaid growth and remodeling creates co-existing instability patterns in contrast to the regular patterns created by growth alone. Constant-stiffness folding with $\mu_{\mathrm{m}} / \mu_{\mathrm{s}}=10,100$, and 1000 generates $\mathrm{n}=7,5$, and 3 initial folds (right). When gradually increasing the stiffness ratio from $\mu_{\mathrm{m}} / \mu_{\mathrm{s}}=10$ to 100 , the initial configuration with sixteen smaller folds is overlaid by four larger folds, $\mathrm{n}=16,4$ (blue). When increasing the stiffness ratio from $\mu_{\mathrm{m}} / \mu_{\mathrm{s}}=100$ to 1000 , fifteen initial folds appear, which then collapse inward on each other as they are dominated by two larger folds, $\mathrm{n}=15,2$ (green). Increasing the stiffness ratio from $\mu_{\mathrm{m}} / \mu_{\mathrm{s}}=10$ to 1000 results in thirteen initial folds superposed by two larger folds, $\mathrm{n}=13,2$ (red).

The rate at which the airway stiffens impacts the primary and secondary failure mode. When the stiffness contrast increases from 10 to 100 (blue), it does so at a slower rate than when it increases from 10 to 1000 (red). The stiffness value inversely impacts the fold number. For the same growth value during the first bifurcation, the blue cylinder is at a lower stiffness than the red, and it folds into $n=16$ folds instead of $n=13$. As the mucosal lining stiffens further, red again does so at a faster rate than the blue, leading to a higher stiffness value when the next bifurcation occurs. This results in fewer folds $\mathrm{n}=2$ for the red cylinder compared to the $\mathrm{n}=4$ folds for the blue cylinder. A faster stiffening rate reaches a larger stiffness contrast sooner and the subsequent bifurcation generates fewer folds in comparison to a system with a slower stiffening rate.

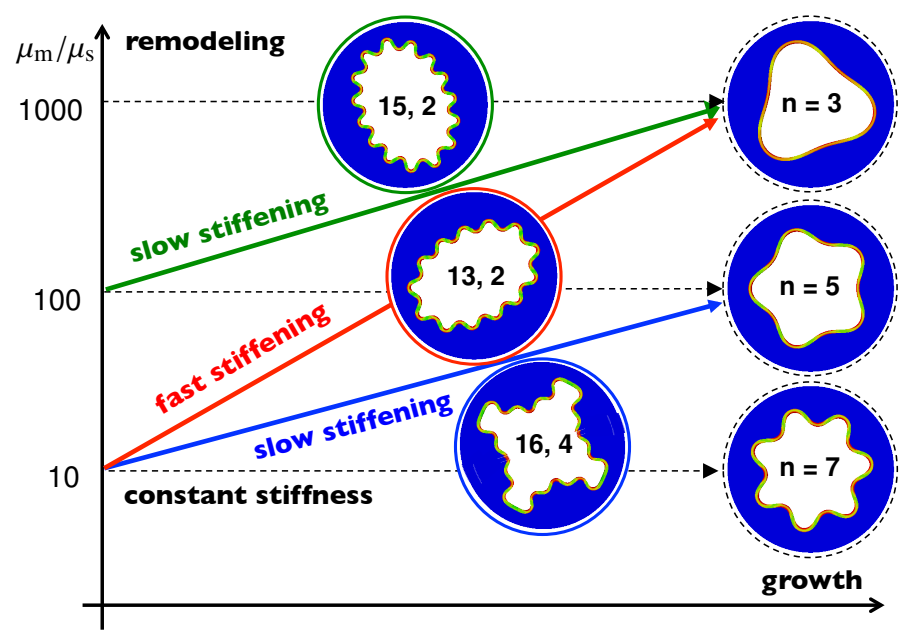

Figure 6: Stiffening rates impact secondary bifurcation. Comparison of regular, constant-stiffness instabilities (dotted lines) and irregular, evolving-stiffness instabilities created by linearly increasing the stiffness contrast from initial to final values (colored solid lines). Altering the stiffness ratio as the inner layer grows causes the initial solution to bifurcate again into a superposed mode with fewer folds corresponding to larger stiffness contrast. 


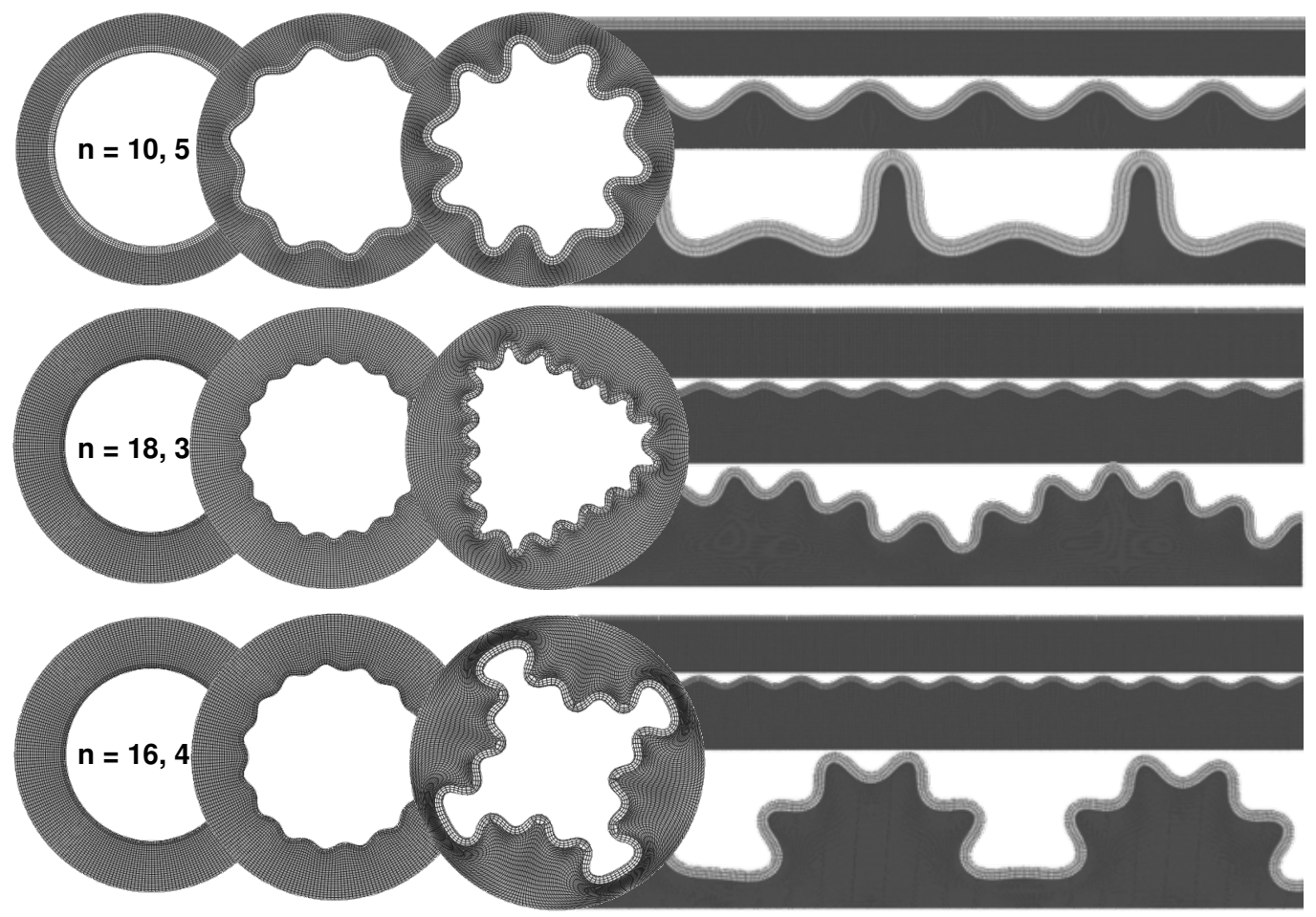

Figure 7: Co-existing instability modes in response to growth and remodeling in circular and rectangular geometries. Upon gradual stiffening, rectangular bi-layered geometries with identical parameters reveal similar instability patterns as circular counterparts. The undeformed configurations initially bifurcate into regular folding patterns and, upon continuing growth and stiffening, instead of increasing the amplitudes of existing folds, new larger folds emerge. Larger folds are lined with smaller folds as each stiffness parameter corresponds to a preferred instability associated with the thickness and stiffness contrast at the onset of folding. For $t_{\mathrm{m}} / R=0.07, t_{\mathrm{S}} / R=0.3, \mu_{\mathrm{m}} / \mu_{\mathrm{s}}=40 \rightarrow 200$, the first bifurcation wavelength $\lambda_{I}=0.84$ occurs at a stiffness ratio of $\mu_{\mathrm{m}} / \mu_{\mathrm{S}}, I=48.8$ and the subsequent bifurcation at $\lambda_{I I}=1.64$ and $\mu_{\mathrm{m}} / \mu_{\mathrm{s}}, I=84.0$ (top). For $t_{\mathrm{m}} / R=0.05, t_{\mathrm{s}} / R=0.5$, and $\mu_{\mathrm{m}} / \mu_{\mathrm{s}}=100 \rightarrow 1000$, the first bifurcation wavelength $\lambda_{I}=0.44$ occurs at a stiffness ratio of $\mu_{\mathrm{m}} / \mu_{\mathrm{s}, I}=147.7$ and the subsequent bifurcation at $\lambda_{I I}=2.75$ and $\mu_{\mathrm{m}} / \mu_{\mathrm{s}, I I}=399.7$ (middle). For $t_{\mathrm{m}} / R=0.04, t_{\mathrm{s}} / R=0.5$, and $\mu_{\mathrm{m}} / \mu_{\mathrm{s}}=100 \rightarrow 1000$, the first bifurcation wavelength $\lambda_{I}=0.40$ occurs at a stiffness ratio of $\mu_{\mathrm{m}} / \mu_{\mathrm{s}, I}=138.7$ and the subsequent bifurcation at $\lambda_{I I}=1.94$ and $\mu_{\mathrm{m}} / \mu_{\mathrm{s}}, I I=197.2$ (bottom).

\subsection{Co-existing instability modes in different geometries}

To explore whether the multiple bifurcations are solely a result of the circular geometry, we performed comparisons with simulations on a rectangular domain. Figure 7 illustrates how we matched the thicknesses of the growing layer $t_{\mathrm{m}} / R$ and the substrate $t_{\mathrm{s}} / R$ with their circular counterparts and unrolled the circumference to derive the length of the rectangle. For the same growth and stiffening rates, we observed similar coexisting instability modes for both circular and rectangular geometries. After an initial small-wavelength instability, a secondary large-wavelength instability emerges. We recorded the wavelengths and stiffness contrasts at which these bifurcations occur in the rectangular geometry. In the first example in Figure 7 , five dominant folds are superposed on ten folds after the second bifurcation emerges, $\mathrm{n}=10,5$ (top). The doubling of the wavelength of the circular geometry is identically reproduced by the rectangular geometry. The second and third example in Figure 7 differ by the initial thickness of the mucosal layer $t_{\mathrm{m}} / R$. The second example (middle) reveals how a thinner mucosal layer results in shorter wavelengths and $n=18,3$ folds in comparison to the third example (bottom) with slightly longer wavelengths and $n=16,4$ folds. For similar stiffening rates, the inverse relationship between fold number and stiffness appears to dictate the subsequent instability mode: the second bifurcation has a larger wavelength for the second example than for the third example.

\subsection{Regional stresses in post-buckling regime}

Figure 8 highlights the evolution of stresses profiles in different regions along the circumference for the $n=10,5$ (left) and $n=18,3$ (right) instabilities from Figure 7. Stresses are normalized by the current stiffness ratio $\mu_{s \mathrm{~m}} / \mu_{\mathrm{s}}$ to allow a oneto-one comparison with non-evolving stiffness ratios. Initially, before the first bifurcation point, the local stress profiles are similar and independent of location. All stresses increase linearly with increasing growth. Beyond the first instability point, the stress at the inward-folding locations drops, while the stress at the outward-folding locations increases. At the second bifurcation point, a new failure mode emerges on top of the initial mode and the local stresses drop and increase as the two modes compete. The emerging stress profiles in Figure 8 explain the changes in folding trajectories, as visually highlighted in Figures 4 to 7 . These changes in trajectories mark the bifurcation points of pattern formation and underline the complex energetic landscape of superposed airway wall growth and remodeling.

Prior to buckling, the local stress increases linearly with increasing growth. Upon primary folding, neighboring regions experience a sudden non-linear increase or decrease in stress. The two adjacent regions continue to have identical non-linear stress trajectories until the subsequent bifurcation emerges. Upon secondary folding, the divergence from the joint stress trajectory characterizes the second instability mode. Differences between the $\mathrm{n}=10,5$ and $\mathrm{n}=18,3$ mode examples indicate that a region that initially prefers a lower stress trajectory at the first bifurcation point can become both the site of a 

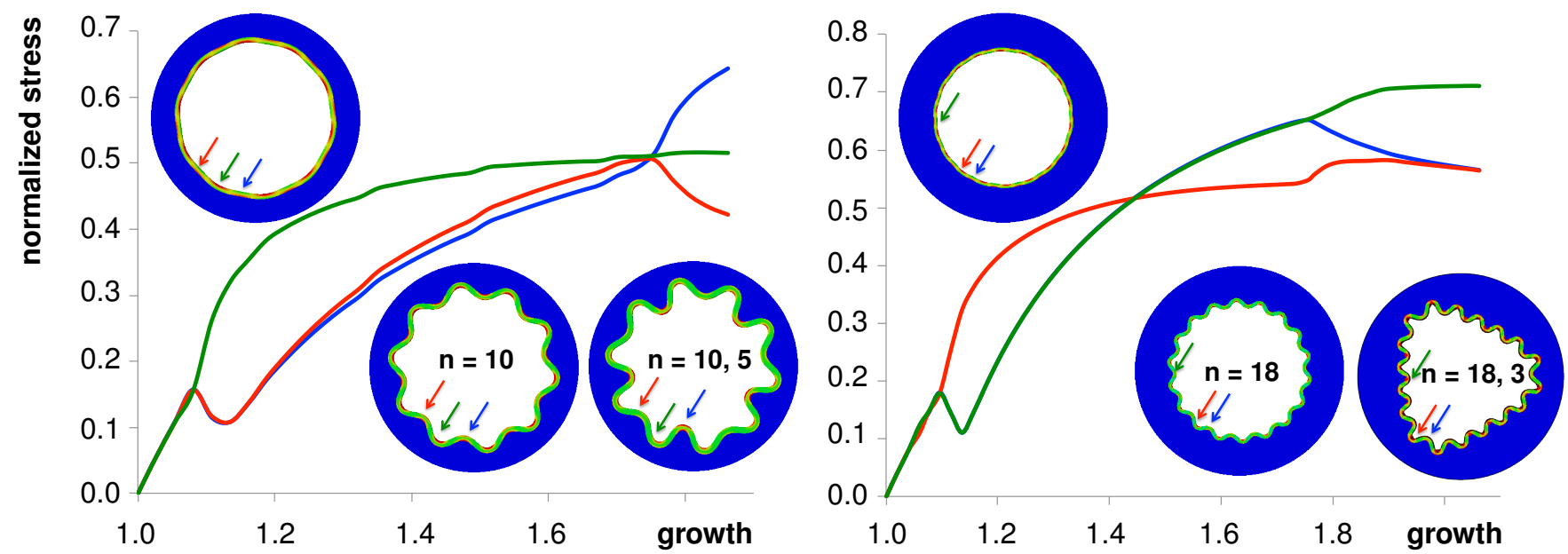

Figure 8: Normalized stress as a function of growth. Regional stress distribution is color-coded by surface location indicated by red, green, and blue arrow. Stress values, normalized by the current stiffness ratio $\mu_{\mathrm{m}} / \mu_{\mathrm{s}}$, are initially identical and increase gradually as a function of growth until the first bifurcation point at which the stress at the inward-folding locations drops, while the stress at the outward-folding locations increase. At the second bifurcation point, a new failure mode emerges on top of the initial mode and the local stresses drop and increase as the two modes compete.

higher or lower stress trajectory thereafter. Regardless of the first bifurcation, upon the onset of the next bifurcation, regions are not bounded by their initial bifurcation. This is in strong contrast to the phenomenon of period doubling, where the secondary bifurcation mode emerges naturally upon growth alone, but is predefined to double the wavelength of the initial instability mode [13].

\subsection{Perturbation insensitivity}

Figure 9 demonstrates that our simulations are truly insensitive to the perturbation pattern. In four different simulations with two random perturbations, p1 and p2, our computational simulation generates similar primary instability patterns and similar superposed secondary instability patterns. The resulting instability patterns are qualitatively comparable and perturbation insensitive.

\section{Discussion}

The objective of this study was to explore the effects of combined growth and remodeling [65], which manifest themselvesin the context of the airway-as inflammation-induced volume increase and an elastosis-induced stiffness change. For various geometries and rates of stiffening, our growth and remodeling simulations predict multiple co-existing instability patterns: The initial folding pattern consists of multiple regular small folds induced by constrained growth. Upon continuing growth, superposed with gradual stiffening, larger secondary folds emerge and compete with the initial folding pattern to generate an irregularly folded inner airway wall lining. Shortand long-wavelength modes co-exist simultaneously. The overall surface morphology agrees with histological cross-sections of the diseased airway wall, in which short and long wavelengths co-exist and ultimately fold inward to cause chronic airway obstruction $[18,30]$.

The initial failure mode corresponds to a lower stiffness ratio between mucosa and submucosa, and the subsequent fewer
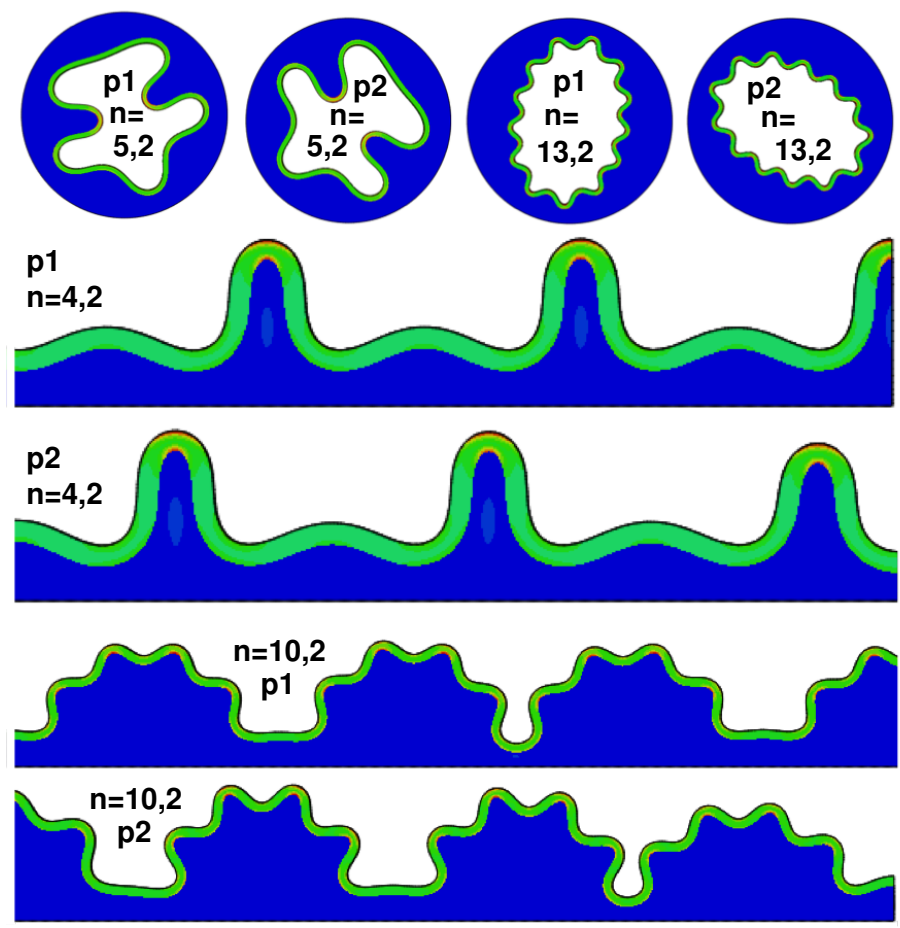

Figure 9: Perturbation insensitivity of the simulation. Four different simulations with two random perturbations, $\mathrm{p} 1$ and $\mathrm{p} 2$, generate similar primary instability patterns and similar superposed secondary instability patterns. The simulated instabilities are perturbation insensitive.

mode configuration are a natural consequence of a gradual increase of this stiffness ratio. Stiffening of the mucosal layer, superposed onto its continuing growth prevents the initial folding mode to stably continue to fold inwards and eventually form contact between neighboring folds. The increasing mucosal stiffness triggers a secondary bifurcation with superposed larger folds, a configuration which is energetically favorable over the pure increase in folding amplitude within the initial folding pattern. 
Figure 7 confirms that the double bifurcation is not exclusive to circular geometries-the shapes of the initial and secondary instability modes in circular geometries were nearly identical to their unfurled rectangular counterparts. Matching the circular geometry with their rectangular equivalents allows us measure and compare the wavelengths between different parameterizations. Our results suggest that the established relationship between the third root of the stiffness contrast and the number of folds still governs the secondary bifurcation as the stiffness contrast evolves $[10,11,43,44]$. The results agree with previous constant-stiffness analyses [33, 46, 67]: For smaller stiffness contrasts, we expect numerous small folds; for larger stiffness contrasts, we expect fewer large folds [23, 45, 68]. Since the stiffness increases beyond the first instability point, subsequent folds are of greater wavelength, which agrees with the expected behavior known from the constant-stiffness case. For the inverse case with an initially higher stiffnesses and gradual softening, we would expect to see fewer initial folds transforming into a multiple-fold configuration. We will consider the case of remodeling-induced softening in future works; however, diseased, scarred, or injured tissues tend to undergo increased stiffening [47, 50, 64], as even seen in aortic aneurysms, which complicate obstruction [59]. Growth of aneurysms, on the contrary, is more likely to become unstable in elastin-deficient, softer vessels, whereas stiffened vessels have the tendency to stabilize the growth process $[61,72]$. Here, we have initially limited the scope of our analysis to remodeling-induced stiffening.

We find that in addition to the non-dimensionalized parameters $t_{\mathrm{m}} / R, t_{\mathrm{s}} / R$, and $\mu_{\mathrm{m}} / \mu_{\mathrm{s}}$, the stiffening rate, or rather the ratio between stiffening rate and growth rate, also impacts the folding pattern. Within a given time period, fast stiffening results in a larger stiffness ratio and consequently triggers larger wavelengths. This trend is clinically relevant: If a patient's health swiftly declines and there is more deterioration of elastin fibers in the airway wall, few-fold configurations with a higher potential of collapse become the more likely failure mode. While two-fold failure modes are not observed in linear buckling analysis of previous studies [7, 45, 68], the airway's ability to form secondary long-wavelength buckling modes to obstruct the lumen, similar to the ones seen in elastosis, sheds light on the potential dangers of airway wall remodeling.

The regional stress development of selected circular geometries in Figure 8 illustrates the linear relation between stress and growth until the initial bifurcation point. The findings from many computational simulations of various geometric and material parameters depicted a linear dependency between critical stress at the onset of primary buckling and the critical stiffness ratio. This agrees with Timoshenko's analytical solution of a thin long beam buckling under an axial load adapted to our growing circular geometry [66]. Varying $t_{\mathrm{m}} / R$ and $t_{\mathrm{s}} / R$ reveals non-linear relationships with the critical stress. These findings for the primary buckling mode suggest that for our circular geometry, the critical stress is linearly related to the stiffness ratio, non-linearly related to the mucosal thickness, and inversely non-linearly related to the thickness of the submucosa. These findings agree with analytical results of infinite rectangular ge- ometries [11].

To understand whether the secondary bifurcation is a result of continuing growth of the inner layer or a consequence of combined growth and stiffening, we illustrate energetic considerations in Figure 8, and compare the normalized stresses of two different folding patterns before and after the first and second bifurcation. Upon emergence of the initial folding pattern, we hold the stiffness contrast constant as the mucosa continues to grow. While a second bifurcation emerges when the airway wall undergoes progressive stiffening, no such secondary folding occurs under constant-stiffness conditions. Extensive continuing growth could eventually trigger a secondary instability in the form of period doubling; however, continuing growth alone cannot explain the emergence of superposed folding patterns with random wavelengths. The second instability we observe in our simulations is a genuine result of the temporally increasing stiffness, and not just an after-effect of continuing growth. It is likely that elastosis could even trigger a conceptually similar third bifurcation, beyond the first and second instability point, associated with even larger stiffness contrasts and larger wavelengths.

Taken together, our results suggest that our secondary bifurcation is not merely a doubling or tripling of the wavelength, as observed in prior studies $[12,13]$. Our secondary bifurcation can be a much greater multiple of the initial wavelength, e.g., by a factor two in the $\mathrm{n}=10,5$ mode, by a factor six in the $\mathrm{n}=18,3$ mode, or by a factor four in the $\mathrm{n}=16,4$ mode in Figure 7. Importantly, all our subsequent patterns arise naturally, and are not triggered by imposed perturbations. Our emerging bifurcation patterns are exclusively triggered by simultaneous inflammation-induced growth and elastosis-induced remodeling.

In exploring a wide variety of geometries subject to evolving stiffness, we find that while the first folding configuration could span as many as 20 folds, the second bifurcation was two, three, four, or five folds. Despite an initial release of energy through buckling, the continued growth of the inner layer does not simply push the folds inwards; rather, the combined stiffening and growth further increases the energy and triggers yet another buckling mode. The initial folding pattern is always overlaid by a stiffer fewer-fold configuration with, in our case, no more than five folds. Studies have shown that a few larger folds are more critical to block the lumen than many smaller folds [22]. Emerging secondary modes with few large folds create a new potential for airway obstruction that had previously been ignored. While clinicians debate the extent to which elastosis and fragmentation remodel the airway wall [49], this model provides insight into the critical role of secondary folding and highlights the potential for elastosis as an important mechanism for airway obstruction and airway collapse.

\section{Concluding remarks}

The objective of this work was to explore the effect of superposed airway wall growth and remodeling, where growth is attributed to airway inflammation and a local influx of cells and remodeling is attributed to airway elastosis and local changes in 
tissue microstructure. Our studies show that combining growth and remodeling creates multiple co-existing instability patterns that can be far more complex than their constant-stiffness counterparts.

We discovered that continuing growth, overlaid by stiffening, triggers the generation of subsequent folding patterns beyond the first instability mode. Elastosis creates superposed secondary instability patterns, which naturally corresponded to the altered stiffness ratio at which they occur. Here, we have accounted for changes in material properties through a computational evolutionary stiffness model. Analytical solutions for these phenomena are nonexistent; our simulations yield valuable insight into complex folding phenomena that have been observed clinically via histological stains, but have not been explained mechanistically to date.

Much of the simplifying assumptions and limitations in this study are due to the lack of clinical data that quantify stiffening during airway elastosis. While physicians have observed a loss of compliance of more than $50 \%$ in specific diseased airway locations, their tools are limited to measuring airway compliance in patients and inherently limited to macroscopic properties [15]. Clinical methods like forced expiration cannot distinguish between mucosal and submucosal layers and do not provide a connection between stiffness and histology of elastin fiber [49]. Here we virtually increased the stiffness over time by orders of magnitude to explore whether stiffness variations could affect surface morphologies. For the lack of better knowledge, we have modeled the airway wall as isotropic NeoHookean. In reality, the airway wall could very well be strain stiffening and anisotropic, and accounting for these phenomena could intensify secondary instability patterns. We are currently characterizing the mechanical properties of the airway wall experimentally to address these phenomena and make our airway wall models more physiological. Another important next step would be to fine-tune the mechanism and calibrate the model by clinical data to investigate whether those findings are significant in airway wall obstruction.

Overall, our results suggest that the mechanism of airway wall stiffening-in addition to growth-plays a critical role in airway obstruction: it not only creates multiple co-existing instability patterns that match histological observations more closely, but it also predicts morphologies that are more likely to generate airway obstruction than growth alone.

\section{Acknowledgments}

We thank Dr. Gerald Berry, Professor of Pathology at the Stanford University Medical Center, for the histology images. This study was supported by the National Science Foundation Graduate Research Fellowship, by the Stanford Science and Engineering Graduate Fellowship, and by the DARE Doctoral Fellowship to Mona Eskandari, and by the National Institutes of Health grant U01 HL119578 to Ellen Kuhl.

\section{References}

[1] Abaqus 6.13. Analysis User's Manual. 2013. Simulia. Dassault Systèmes.
[2] Ambrosi, D.; Ateshian, G.A.; Arruda, E.M.; Cowin, S.C.; Dumais, J; Goriely, A.; Holzapfel, G.A.; Humphrey, J.D.; Kemkemer, R.; Kuhl, E.; Olberding, J.E.; Taber, L.A.; Garikipati, K. Perspectives on biological growth and remodeling. J Mech Phys Solids. 2011, 59, 863-883.

[3] Asthma Statistics. Asthma mortality rates have been steadily increasing since the 1970s. 2013, http:// www.achooallergy.com/asthma- statistics.asp

[4] Bai, T.R.; Knight, D.A. Structural changes in the airways in asthma: observations and consequences. Clinical Science. 2005, 108, 463-477.

[5] Bai, A.; Eidel, D.H.; Hogg, J.C.; James, A.L.; Lambert, R.K.; Ludwig, M.S.; Martin, M.; McDonald, D.M.; Mitzner, W.A.; Okazawa, M.; Pack, R.J.; Paré,P.D.; Schellenberg, R.R.; Tiddens, H.A.; Wagner, E.M.; Yager, D. Proposed nomenclature for quantifying subdivisions of the bronchial wall. J Appl Physiol. 1994, 77, 1011-1014.

[6] Balbi, V.; Ciarletta, P. Morpho-elasticity of intestinal villi. J Roy Soc Interface. 2013, 10, 20130109.

[7] Balbi, V; Kuhl, E; Ciarletta, P. Morphoelastic control of gastro-intestinal organogenesis: theoretical predictions and numerical insights. $J$ Mech Phys Solids. 2015, 78, 493-510.

[8] Barnett S.B.; Nurmagambetov T.A.; Costs of asthma in the United States: 2002-2007. J Allergy Clin Immunol. 2011, 127, 145-152.

[9] Bergeron, C.; Al-Ramli, W.; Hamid, Q. Remodeling in asthma Proc Am Thorac Soc. 2009, 6, 301-305.

[10] Budday, S.; Steinmann, P.; Kuhl, E. The role of mechanics during brain development. J Mech Phys Solids. 2014, 72, 75-92.

[11] Budday, S; Raybaud, C; Kuhl, E. A mechanical model predicts morphological abnormalities in the developing human brain. Sci Rep. 2014, 4, 5644.

[12] Budday, S; Steinmann, P; Kuhl, E. Secondary instabilities modulate cortical complexity in the mammalian brain. Phil Mag. 2015, 95, 3244-3256.

[13] Budday, S; Kuhl, E; Hutchinson, J.W. Period-doubling and period-tripling in growing bilayered systems. Phil Mag. 2015, 95, 3208-3224.

[14] Bousquet, J.; Jeffery, P. Asthma from bronchoconstriction to airways inflammation and remodeling. Am J Respir Crit Care Med. 2000, 161, 1720-1745.

[15] Brackel, H.J.; Pedersen, O.F.; Mulder, P.G.; Overbeek, S.E.; Kerrebijn, K.F.; Bogaard, J. M. Central airways behave more stiffly during forced expiration in patients with asthma. Am J Respir Crit Care Med. 2000, 162, 896-904.

[16] Bramley, A.M.; Thomson, R.J.; Roberts, C.R.; Schellenberg, R.R. Hypothesis: excessive bronchoconstriction in asthma. Europ Respir J. 1994, 7, 337-341.

[17] Cao, Y.P.; Li, B.; Feng, X.Q. Surface wrinkling and folding of core-shell soft cylinders. Soft Matter. 2012, 8, 556-562.

[18] Carroll, N.G.; Perry, S.; Karkhanis, A.; Harji, S.; Butt, J.; James, A.L.; Green, F.H.Y. The airway longitudinal elastic fiber network and mucosal folding in patients with asthma. Am J Respir Crit Care Med. 2000, 161, 244-248.

[19] Ciarletta, P,; Ben Amar, M. Growth instabilities and folding in tubular organs: A variational method in non-linear elasticity. Int J Non-Lin Mech. 2012, 47, 248-257.

[20] Ciarletta, P.; Balbi, V.; Kuhl E. Pattern selection in growing tubular tissues. Phys Rev Lett. 2014, 113, 248101.

[21] Cohn, L.; Elias, J.A.; Chupp, G.L. Asthma: Mechanisms of disease persistence and progression. Annu Rev Immunol., 2004, 22, 789-815.

[22] Eskandari M.; Pfaller M.R.; Kuhl E. On the role of mechanics in chronic lung disease. Materials. 2013, 6, 5639-5658.

[23] Eskandari, M; Kuschner, W.G; Kuhl, E. Patient-specific airway wall remodeling in chronic lung disease. Ann Biomed Eng. 2015, 10, 2538-2551.

[24] Eskandari, M; Kuhl, E. Systems biology and mechanics of growth. WIREs Syst Biol Med. 2015, 7, 401-412.

[25] Garikipati, K. The kinematics of biological growth. Appl Mech Rev. 2009, 62,030801 .

[26] Genet, M.; Lee, L.C.; Baillargeon, B.; Guccione, J.M.; Kuhl, E. Modeling pathologies of systolic and diastolic heart failure. Ann Biomed Eng. 2016, 44, 112-127.

[27] Göktepe, S.; Abilez, O.J.; Parker, K.K.; Kuhl, E. A multiscale model for eccentric and concentric cardiac growth through sarcomerogenesis. $J$ Theor Bio. 2010, 265, 433-442.

[28] Himpel, G.; Kuhl, E.; Menzel, A.; Steinmann, P. Computational modeling of isotropic multiplicative growth. Comp Mod Eng Sci. 2005, 8, 119-134. 
[29] Hogg, J.C. Pathophysiology of airflow limitation in chronic obstructive pulmonary disease. Lancet. 2004, 364, 709-721.

[30] Hogg, J.C.; Chu, F.; Utokaparch, S.; Woods, R.; Elliott, W.M.; Buzatu, L.; Cherniack, R.M.; Rogers, R.M.; Sciurba, F.C.; Coxson, H.O.; Pare, P.D The nature of small-airway obstruction in chronic obstructive pulmonary disease. New Engl J Med. 2004, 350, 2645-2653.

[31] Holzapfel, G.A. Nonlinear solid mechanics: a continuum approach for engineering. John Wiley and Sons Ltd., 2000.

[32] Holland, M. A.; Miller, K. E.; Kuhl, E. Emerging brain morphologies from axonal elongation. Ann Biomed Eng. 2014, 1-14.

[33] Hrousis, C.A.; Wiggs B.R.; Drazen J.M.; Parks D.M.; Kamm R.D.; Mucosal Folding in Biologic Vessels. Journal of Biomech Eng. 2002, 124, 334-341.

[34] James, A.L.; Paré, P.D.; Hogg, J.C.; The mechanics of airway narrowing in asthma. Am Rev Respir Disease. 1989, 139, 242-246.

[35] Javili, A; Dortdivanlioglu, B; Kuhl, E; Linder, C. Computational aspects of growth-induced instabilities. Comp Mech. 2015, 56, 405-420.

[36] Jia, F; Li, B; Cao, Y.P.; Xie, W.H.; Feng, X.Q. Wrinkling pattern evolution of cylindrical biological tissues with differential growth. Physical Review E. 2015, 91, 012403. (2015).

[37] Jin, L.; Cai, S.; Suo, Z. Creases in soft tissues generated by growth. EPL Frontiers of Physics. 2011, 95, 64002.1-64002.6.

[38] Kamm, R.D. Airway wall mechanics. Ann Rev Biomed Eng. 1999, 1, 47 72

[39] Kuhl E. 2014. Growing matter - A review of growth in living systems. J Mech Behavior Biomed Mat. 29:529-543.

[40] Kumar, R.K.; Understanding airway wall remodeling in asthma: a basis for improvements in therapy. Pharmacol Ther. 2001, 91, 93-104.

[41] Kuwano, K.; Bosken, C.H.; Paré, P.D.; Bai, T.R.; Wiggs, B.R.; Hogg. J.C. Small airways dimensions in asthma and in chronic obstructive pulmonary disease. Am Rev Respir Disease. 1993, 148, 1220-1225.

[42] Lambert, R.K. Role of bronchial basement membrane in airway collapse. J Appl Phys. 1991, 71, 666-673.

[43] Lejeune, E.; Javili, A.; Linder, C. Understanding geometric instabilities in thin films via a multi-layer model. Soft Matter. 2016, 12, 806-816.

[44] Lejeune, E.; Javili, A.; Linder, An algorithmic approach to multi-layer wrinkling. Extr Mech Lett. 2016, 7, 10-17.

[45] Li, B.; Cao, Y.P.; Feng, X.Q.; Gao, H. Surface wrinkling of mucosa induced by volumetric growth: Theory, simulation and experiment. $J$ Mech Phys Solids. 2011, 59, 758-774.

[46] Li, B.; Cao, Y.P.; Feng, X.Q.; Gao, H. Mechanics of morphological instabilities and surface wrinkling in soft materials: A review. Soft Matter. 2012, 8, 5728-5745.

[47] Lu Y.Y.; Chen, Y.C.; Kao, Y.H.; Wu, T.J.; Chen, S.A.; Chen, Y.J. Extracellular matrix of collagen modulates intracellular calcium469handing and electrophysiological characteristics of HL-1 cardiomyocytes with activation of angiotensin II type 1 receptor. J Card Fail. 2011, 17, 82-90

[48] Martin, J.G.; Duguet, A.; Eidelman, D.H. The contribution of airway smooth muscle to airway narrowing and airway hyperresponsiveness in disease. Eu Respir J. 2000, 16, 349-354.

[49] Mauad, T.; Xavier, A. Elastosis and fragmentation of fibers of the elastic system in fatal asthma. Am J Respir Crit Care Med. 1999, 160, 968-975.

[50] Maurice, D.M.; The structure and transparency of the cornea. J Physiol. 1957, $136263 ? 286$

[51] Menzel, A. A fibre reorientation model for orthotropic multiplicative growth. Biomech Model Mechanobio. 2007, 6, 303-320.

[52] Menzel, A.; Kuhl, E. Frontiers in growth and remodeling. Mech Res Comm. 2012, 42, 1-14.

[53] Moulton, D.E.; Goriely, A. Circumferential buckling instability of a growing cylindrical tube. J Mech Phys Solids. 2011, 59, 525-537.

[54] Moulton, D.E.; Goriely, A. Possible role of differential growth in airway wall remodeling in asthma. $J$ Appl Physiol. 2011, 110, 1003-1012.

[55] National Institutes of Health. Survey: Awareness of COPD the Nation's Fourth Leading Cause of Death is Rising, but Understanding is Still Low: News Release. U.S National Library of Medicine. 2009 http://www.nih.gov/news/health/nov2009/ nhlbi- 02.htm

[56] Papastavrou, A.; Steinmann, P.; Kuhl, E. On the mechanics of continua with boundary energies and growing surfaces. J Mech Phys Solids. 2013, 61, 1446-1463.

[57] Pfaller M. Instabilities during mucosal folding induced by volumetric growth. Masters Thesis $\mathbf{2 0 1 2}$.
[58] Politi, A. Z.; Donovan, G. M.; Tawhai, M. H.; Sanderson, M. J.; Lauzon, A. M.; Bates, J. H.; Sneyd, J. A multiscale, spatially distributed model of asthmatic airway hyper-responsiveness. J Theor Bio. 2010, 2664 614-624.

[59] Raaz, U; Zöllner, A.M; Schellinger, I.N; Toh, R; Nakagami, F; Brandt, M; Emrich, F.C.; Kayama, Y; Eken, S; Adam, M; Maegdefessel, L; Hertel, T; Deng, A; Jagger, A; Buerke, M; Dalman, R.L; Spin, J.M; Kuhl, E; Tsao, P.S. Segmental aortic stiffening contributes to experimental abdominal aortic aneurysm development. Circulation. 2015, 131, 1783-1795.

[60] Rodriguez, E.K.; Hoger, A.; McCulloch, A.D. Stress-dependent finite growth in soft elastic tissues. J Biomech. 1994, 27, 455-467.

[61] Satha, G.; Lindstrom S.B.; Klarbring, A. A goal function approach to remodeling of arteries uncovers mechanisms for growth instability. Biomech Model Mechanobiol. 2014, 13, 1243-1259.

[62] Schmid, H.; Pauli, L.; Paulus, A.; Kuhl, E.; Itskov, M. Consistent formulation of the growth process at the kinematic and constitutive level for soft tissues composed of multiple constituents. Comp Meth Biomech Biomed Eng. 2012, 15, 547-561.

[63] Stalhand, J.; Klarbring, A.; Holzapfel, G.A. A mechanochemical 3D continuum model for smooth muscle contraction under finite strains. $J$ Theor Bio. 2011, 268120-130.

[64] Stewart, J.I.; Criner, G.J. The small airways in chronic obstructive pulmonary disease: pathology and effects on disease progression and survival. Curr Opinion Pulm Med. 2013, 19, 109-115.

[65] Taber, L.A. Biomechanics of growth, remodeling, and morphogenesis. Appl Mech Rev. 1995, 48, 487-545.

[66] Timoshenko, S. P.; Gere, J.M. Buckling of shells. In: Theory of Elastic Stability (2nd ed.). Toronto: McGraw-Hill, 1961, chapt. 11, 457-520.

[67] Wiggs, B.R.; Moreno, R.; Hogg, J.C.; Hilliam, C.; Paré, P.D.; A model of the mechanics of airway narrowing. J Appl Physiol. 1990, 69 849-860.

[68] Wiggs, B.R.; Hrousis, C.A.; Drazen, J.M.; Kamm, R.D. On the mechanism of mucosal folding in normal and asthmatic airways. J Appl Physiol. 1997, 83, 1814-1821.

[69] Wilson, L.; Devine, E. B.; So, K. Direct medical costs of chronic obstructive pulmonary disease: chronic bronchitis and emphysema. Respir Med. 2000, 83, 204-213.

[70] Xie, W.H.; Li, B.; Cao, Y.P.; Feng, X.Q. Effects of internal pressure and surface tension on the growth-induced wrinkling of mucosae. J Mech Behavior Biomed Mat. 2014, 29, 594-601.

[71] Yang, L., Li, J., Mo, H., Pidaparti, R. M., Witten, T. M. Possible role of collagen reorientation during airway remodeling on mucosal folding. $J$ Engr Math. 2015, online first; doi:10.1007/s10665-014-9774-6.

[72] Zohdi, T.I.; Holzapfel, G.A.; Berger, S.A. A phenomenological model for atherosclerotic plaque growth and rupture. J Theor Bio. 2004, 227, 437-443.

[73] Zöllner, A.M.; Buganza Tepole, A.; Kuhl, E. On the biomechanics and mechanobiology of growing skin. J Theor Bio. 2012, 297: 166-175. 


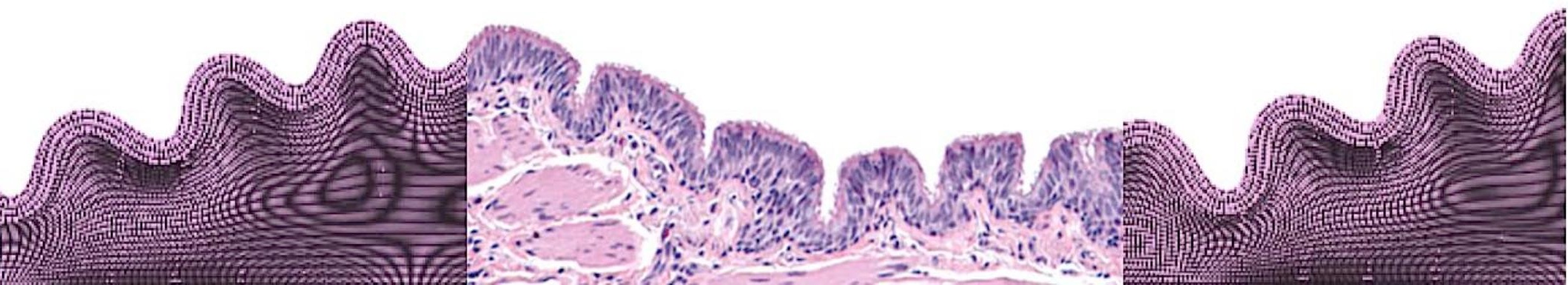

